\title{
Fabrication and Model Simulation of the Ring-shaped Gate Carbon Nanotube Emitter Arrays
}

Jianfeng $\mathrm{Wu}$ and Jun Jiao

Physics Department, Portland State University, P.O Box 751, Portland, OR 97207

The high aspect ratio of the carbon nanotube (CNT) enhances the local electric field close to the nanotube cap [1]. In a previous study, an effective "bottom-up" method was investigated to fabricate plain gate CNT emitter arrays by combining the dual-beam focused ion beam (FIB) technology and plasma enhanced chemical vapor deposition (PECVD) process (Fig. 1a) [2]. The diameter of these CNT emitters is from 30 to $60 \mathrm{~nm}$. A high resolution TEM image of a typical CNT emitter is shown in Fig. 1b. The Ni catalyst is located at the tip of the CNTs. The internal structures of the CNT emitter display bamboo-like fringes characterized by periodic curving graphitic bands normal to the tube axis. During field emission measurement, we found that the capacitance between the plain gate and cathode layer limited high frequency operation. In addition, the larger capacitance could cause current leakage and perhaps break down the $\mathrm{SiO}_{2}$ isolating layer [3]; thus, it is important to keep the capacitance as low as possible. Here we designed a ring-shaped gate (Fig. 2) to replace the plain gate. Computer simulations were also carried out to investigate the effect of the ring-shaped gate on the changes to the local electric field compared with the local electric field from a plain gate device.

To construct the ring-shaped gate device, a multilayer silicon wafer with an embedded catalyst layer was fabricated (the details of each layer are shown in Fig. 3a). For positive PMMA resist, the e-beam exposure will break the bonds of the large PMMA molecular chains, and the smaller chains will be more soluble in the developer than the unbroken (unexposed) chains. After gate coating and PMMA lift-off, we had successfully fabricated a chromium (Cr) round gate (Fig. 3b). A dual-beam FIB was then used to mill arrays of holes to generate the ring-shaped gate and expose the Ni catalyst for further CNT emitter growth (Fig. 3c). Compared with the large capacitance of the plain gate, the ring-shaped gate significantly reduced the gate-cathode capacitance from $138 \mathrm{pF}$ to $3.1 \mathrm{pF}$.

In order to simulate the effect of the ring-shaped gate, we used the finite element method to calculate the variation in electrostatic potential energy and plotted the electric field equipotential lines (represented as a color spectrum in Fig. 4). The local electric field intensity at the CNT tips increased from 4.90 to $5.48 \mathrm{~V} / \mathrm{nm}$ when the gate settings were changed from plain gate to ringshaped gate. In general, the larger the local electric field, the easier it is to initiate the field emission.

In summary, combining the electron beam lithography technique, the dual-beam FIB technique and the PECVD process enables us to effectively synthesize ring-shaped gate field emission devices. The ring-shaped gate design will decrease the capacitance between the gate and cathode. As a result, it will decrease the required power to drive the emitters. Model simulations demonstrate that the equipotential lines and local electric field at the CNT tips are affected by a ring-shaped gate setting. It is expected that the fabrication and simulation results introduced here will provide useful guidance for the design and fabrication of triode-type CNT based field emission device applications [4]. 


\section{References}

[1] W. I. Milne et al., J. Mater. Chem., 14 (2004) 933.

[2] J. Wu et al., Nano Letters., 9(2) (2009) 595.

[3] J. Bird, Electrical and Electronic Principles and Technology, Newnes, Burlington, 2003.

[4] Financial support for this research was provided in part by the NSF under awards No. ECS0348277, ECS-0520891, and DMR-0649280.

Fig. 1a

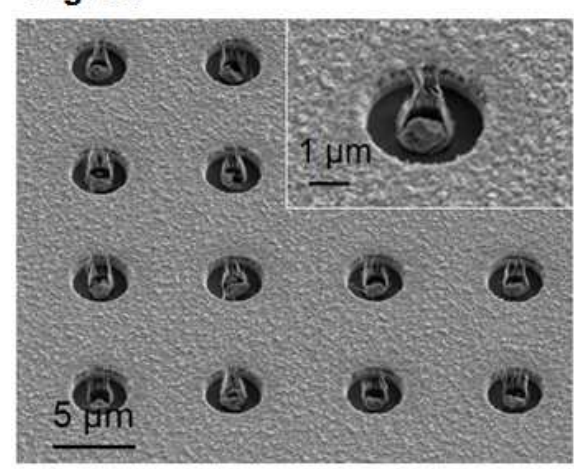

Fig. 3a

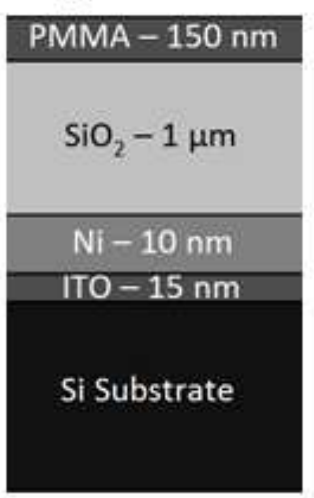

Fig. 1b

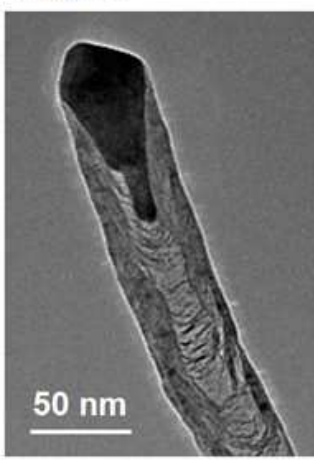

Fig. 2

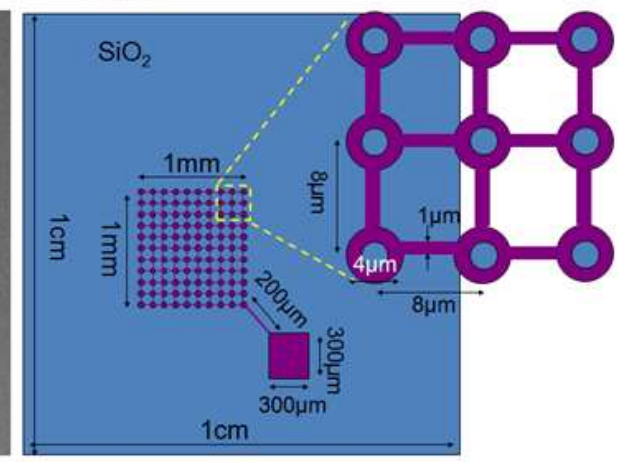

Fig. 3c
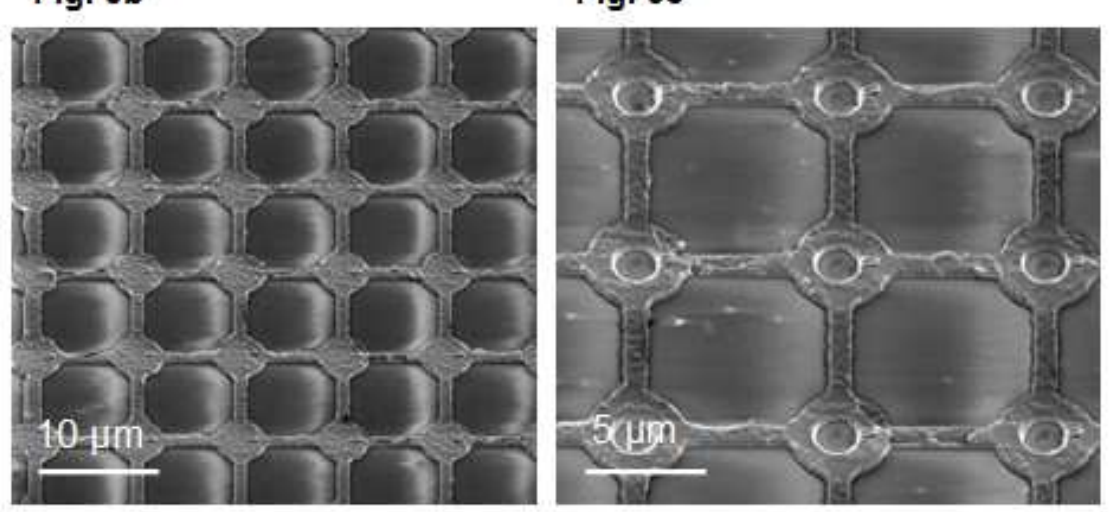

Fig. 4
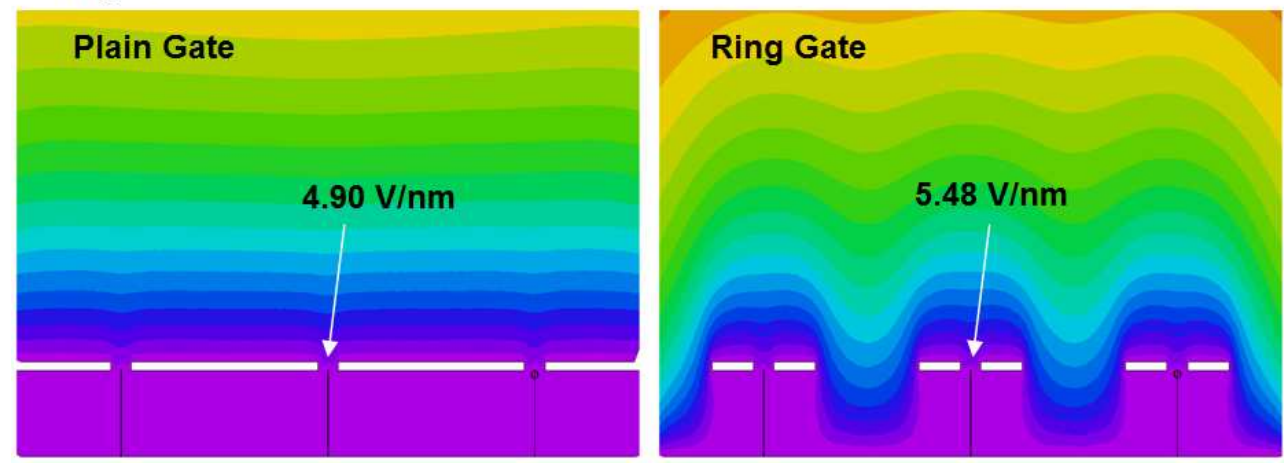

FIG. 1. (a) A triode-type plain gated CNT emitter arrays. (b) TEM image of one of CNTs grown in PECVD. The Ni catalyst is located at the tip of CNT while bamboo-type fringes form inside. FIG. 2. Schematic of ring-shaped gate.

FIG. 3. (a) Schematic of the multilayer substrate for ring-shaped gate fabrication. (b) SEM images of round gate. (c) Mill arrays of holes to generate ring-shaped gate and expose the Ni catalyst for further CNT emitters growth.

FIG. 4. The model simulation equipotential lines are plotted in plain / ring gate settings. 